\title{
The diagnosis and management of severe endometriosis
}

\section{Introduction}

Endometriosis is a common gynecological condition predominantly affecting women in their reproductive years. Estimated prevalence rates are approximately $10 \%$, but in cases of sub fertility can be as high as $25-40 \% .{ }^{1}$ Symptoms vary, but for many women it can be a debilitating condition with a significant impact on their quality of life. ${ }^{2}$ Endometriosis is defined as the presence of endometriotic glands and stroma out-with the uterine cavity. Three types of endometriosis are generally described: peritoneal, ovarian and deep infiltrating endometriosis (DIE), with the latter being defined as the infiltration of endometrial deposits of $\geq 5 \mathrm{~mm}$ into surrounding tissue. ${ }^{3}$ The areas most commonly affected include the uterosacral ligaments (USL), recto-sigmoid colon, recto-vaginal septum (RVS), vagina and bladder. The symptoms of endometriosis are very much dependent on the location of the disease however dysmenorrheal, chronic pelvic pain, deep dyspareunia, fatigue, and sub fertility continue to be the leading symptoms. ${ }^{4}$ DIE involving the RVS is generally associated with more severe forms of dyschezia and dyspareunia ${ }^{5}$ and DIE involving the urinary tract can present with frequency, nocturia, bladder spasms and haematuria. ${ }^{6}$ Delay in the diagnosis of endometriosis still remains a problem, with a reported mean time from initial complaint to diagnosis varying from 7.96 to 11.73 years. ${ }^{7}$ A variety of diagnostic methods have been evaluated over the past few decades in diagnosing DIE, such as transvaginal ultrasound (TVS), magnetic resonance imaging (MRI), transrectal ultrasound, barium enema and colonoscopy, however definitive diagnosis is still often only made at laparoscopy. ${ }^{8-12}$ There is a wide variety of treatment options available ranging from ovarian suppression using hormonal agents to radical surgery. In DIE the efficacy of medical treatment is often suboptimal, with high recurrence rates on cessation of treatment ${ }^{13,14}$ and in such cases many advocate the use of more radical surgical excision. Debate and controversy still exists as to how radical surgery should be when excising DIE and its long-term benefits and complications. This aim of this article is to appraise the current evidence for the diagnosis of DIE as well as the various different surgical options available and to discuss their benefits, risks and limitations.

\section{Role of BSGE/tertiary referral centres}

All cases where DIE has been previously diagnosed or there is a strong diagnostic suspicion should be referred to a tertiary referral centre for assessment. The laparoscopic management of such cases can be technically challenging and assessment by a multidisciplinary team with the necessary skills, expertise and experience to manage such complex cases is essential. Ideally the multidisciplinary team should include gynecological, colorectal and urological surgeons, as well as a specialist pain management team and a specialist endometriosis nurse.

\section{Pre-operative assessment}

Thorough assessment and preoperative planning is essential in these complex cases. Firstly full staging is imperative to allow thorough patient counseling as to the extent of the radical surgery and potential short-term and long-term complications. Secondly it is essential to ensure adequate surgical time is allocated and the

\author{
Volume 4 Issue 5 - 2017
}

Rebecca Mallick
Western Sussex Hospital, NHS Foundation Trust, UK

Correspondence: Rebecca Mallick, Western Sussex Hospital, NHS Foundation Trust, St Richards Hospital, Chichester, UK, Tel 074-II6-17I-43,Email rmallick@doctors.org.uk

Received: March 05, 2017 | Published: August 03, 2017

correct expertise is available especially with regards to colorectal and urological input. Historically staging by means of "first look" laparoscopy often combined with cystoscopy and sigmoidoscopy were considered to be the gold standard to fully assess bladder and bowel involvement and plan further radical surgery. However in cases of an obliterated pelvis, laparoscopic visualization may be suboptimal and the depth of invasion and full extent of the disease may be difficult to assess. The diagnosis of endometriosis on laparoscopy is also highly dependent on the experience and skill of the surgeon performing the procedure; the depth of infiltration of endometrial deposits can often be misjudged and retroperitoneal and vaginal endometriosis often missed ${ }^{4}$ (Figure1). Both TVS and MRI have been found to be highly sensitive in diagnosing and assessing the extent of DIE., ${ }^{9}$ With regards to bowel endometriosis, a systematic review assessing the diagnostic value of TVS as a pre-surgical tool to detect bowel endometriosis was undertaken by Hudelist et al. ${ }^{32}$ and $91 \%$ sensitivity and $98 \%$ specificity was reported. ${ }^{9}$ Thus TVS is a very useful and readily available tool in identifying or ruling out bowel endometriosis and assessing the extent of invasion pre-operatively. However it is very much operator dependent and should only be performed by highly experienced clinicians. ${ }^{4}$ The use of MRI to stage DIE preoperatively is also widely under taken and studies have demonstrated a high accuracy in predicting bowel endometriosis with a sensitivity and specificity of $88 \%$ and $98 \%$ respectively. ${ }^{10,15}$ MRI has also been shown to be useful in detecting DIE involving the USL, vagina, RVS and bladder. ${ }^{15}$ MRI is less operator-dependent, however is slightly less sensitive in detecting bowel endometriosis and overall is a more expensive and less readily available tool when compared to TVS. Barium enema has also been evaluated in the assessment of bowel endometriosis and has shown to be useful with a sensitivity of $88 \%$ and specificity of $93 \%,{ }^{16}$ however when compared to TVS appears to be inferior, ${ }^{17}$ but may be useful in selected cases such as those with high recto-sigmoid or sigmoid lesions. Trans-rectal ultrasonography has also been found to be useful in assessing recto-vaginal endometriosis with a sensitivity and specificity of $96 \%$ and $100 \%$ respectively, however this technique is less useful in assessing more distal lesions such as USL involvement. ${ }^{12}$ Colonoscopy is also only useful in a select group of patients, as it is often negative in cases of small bowel nodules, and only in rare cases of very large nodules with significant bowel obstruction will a colonoscopy be positive. ${ }^{11}$ In all cases of severe endometriosis it is also prudent to fully assess the urinary tract. 
Bladder endometriosis can be diagnosed by TVS, ideally while the patient has a full bladder to facilitate the detection of the more discrete nodules in the bladder wall. ${ }^{18}$ TVS is reported to have a high accuracy $(91-95 \%)$ in the detection of bladder nodules. ${ }^{18,19} \mathrm{MRI}$ is also useful particularly for smaller bladder lesions. ${ }^{20}$ Cystoscopy can also be beneficial to preoperatively assess the size/position of a bladder nodule and it relationship to the ureteric orifices and if indicated a biopsy can be taken for histological diagnosis ${ }^{4}$ (Figure 2). Renal ultrasound, MRI or computerized tomography (CT) urogram is the investigations of choice to assess endometriosis affecting the ureter and the presence of hydronephrosis. In the presence of hydronephrosis, consideration should be given to performing a radioisotope (MAG3 or DMSA) scan to assess renal function. Pre-operatively it is thus recommended that all patients with suspected DIE should have detailed imaging, whether TVS or MRI and renal ultrasound, to assess bowel, ureter and bladder involvement to fully prepare for further management. Further investigations such as barium enema, colonoscopy and radioisotope scans may be required in selected patients. The decision on the type of imaging should be decided by local protocols and expertise, but ideally the approach should be multidisciplinary between surgeons and radiologists.

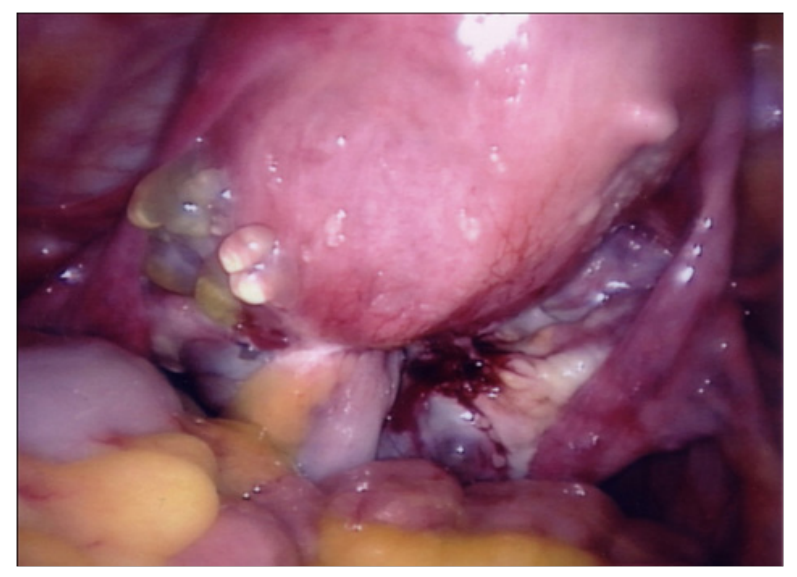

Figure 1 Misjudged and retroperitoneal and vaginal endometriosis.

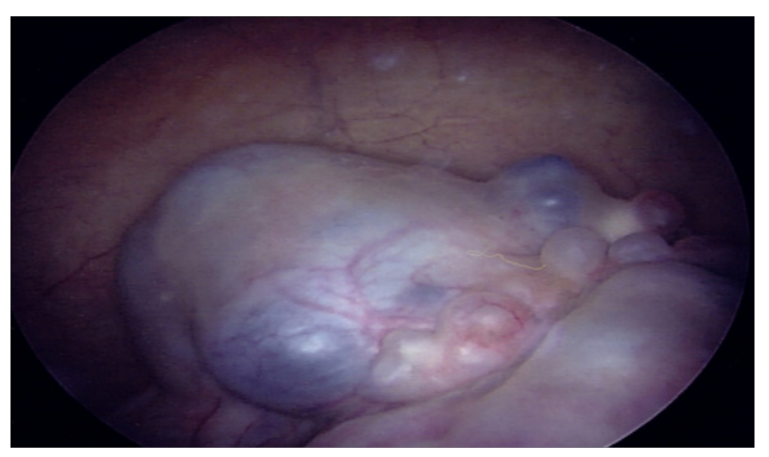

Figure 2 Preoperatively assess the size/position of a bladder nodule.

\section{Bladder and ureteric endometriosis}

DIE involving the urinary tract is uncommon; however its exact prevalence is not entirely know. It is thought to occur in approximately $1-2 \%$ of patients with endometriosis and involves the bladder in $90 \%$ of these cases. ${ }^{18,21}$ Bladder endometriosis can be described as superficial or deep depending on the degree of invasion. Deep bladder endometriosis involves invasion of the muscular is and symptoms can include dysuria, frequency, nocturia, bladder spasms, suprapubic pain and haematuria. ${ }^{18}$ Superficial endometriosis describes endometriosis on the peritoneal surface overlying the bladder.

Endometriosis involving the ureter can either be described as intrinsic or extrinsic, with the latter the more prevalent. In extrinsic cases, the ureter is compressed by the surrounding endometriotic tissue, with resultant fibrosis, hydronephrosis and impaired renal function in up to $30 \%$ of cases $^{6}$ (Figure 3). Intrinsic involvement, defined as endometriotic tissue infiltrating into the ureteral muscular is a rare occurrence. Prior to surgery involving the urinary tract, many surgeons advocate the prophylactic insertion of ureteric catheters/ stents. There is limited evidence in the literature to suggest that stenting actually reduces ureteric injury, ${ }^{22}$ but the use of stents undoubtedly improve visualization of the ureters and hence may make dissection technically easier. They are also particularly useful in duplex urinary systems, where unusual anatomy may increase the risk of injury. Size 6F Stamey catheters may be inserted pre-operatively using an operating cystoscopy and removed at the end of the procedure without the need for radiographic image intensification. The postoperative insertion of double $\mathrm{J}$ stents is indicated if the ureter has been opened and re-anastomosed or re-implanted into the bladder or if there has been extensive ureteriolysis and a potential risk of devascularisation. Alternatively some advocate the primary insertion of double J stents; again this is generally surgeon's preference rather than evidence based. If double $\mathrm{J}$ stents are inserted, they should generally be removed 6 weeks later with a check urogram performed after removal to exclude ureteric leaks. In cases of superficial bladder disease it is often possible to shave the disease off the peritoneal surface of the bladder. However in cases of deep infiltration of the bladder wall full excision is indicated. The bladder should be mobilised first, by opening the uterovesical fold and the dome of the bladder then opened to reveal the extent of the nodule, assessing carefully its relationship to the ureteric orifices. The nodule should then be excised in its entirety and consideration should be given to inserting prophylactic double $\mathrm{J}$ stents particularly if the distance between the border of the lesion and the ureteric orifice is $<2 \mathrm{~cm}^{14}$ (Figure 4). The bladder is closed using a double layer (although many advocate the use of one layer) of interrupted or continuous absorbable sutures such as $2 / 0$ polyglactin and closure checked using methylene blue dye. A free draining catheter should be left in for at least 7days, ideally with a check cystogram to ensure there is no leak prior to removal of the catheter. Laparoscopic partial cystectomy has been found to be a safe and effective treatment for bladder endometriosis in multiple studies with symptomatic improvement in $95-100 \%$ of patient, few complications noted and low recurrence rates. ${ }^{14,21,23}$

With regards to ureteric disease, surgical management of such cases is dependent on numerous factors including patient symptoms, the type of ureteric involvement and also the presence of hydronephrosis/significant strictures In the vast majority of cases the conservative approach with ureteriolysis to mobilize and free the ureterand resection of the surrounding endometriotic tissue and fibrosis may be sufficient. In rare cases where the muscular is involved and or there is significant stenosis, resection of a portion of the ureter with either end-to-end anastomosis or re-implantation with/without a poses hitch may be necessary (Figure 5). Choice of technique is dependent of the location and size of the resected ureteric segment, ${ }^{6}$ however both techniques have been successfully described 
in the literature with significant improvement in symptoms and low complication and recurrence rates. ${ }^{21,24}$ In very rare cases renal function can be significantly reduced in the presence of severe hydronephrosis and in such cases consideration should be given to nephrectomy due to the risks of recurrent infection and vascular hypertension. This can be performed laparoscopically in a multidisciplinary setting at the time of the primary surgery for endometriosis..$^{25,26}$

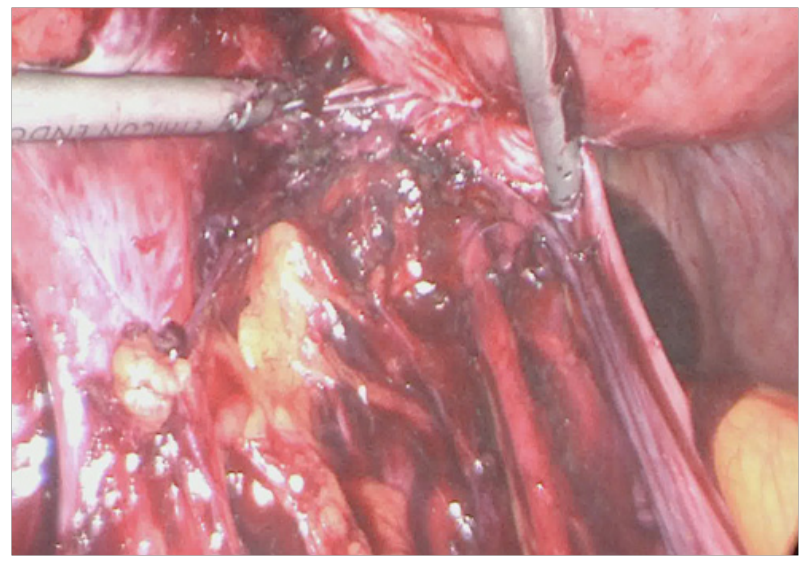

Figure 3 Resultant fibrosis, hydronephrosis and impaired renal function.

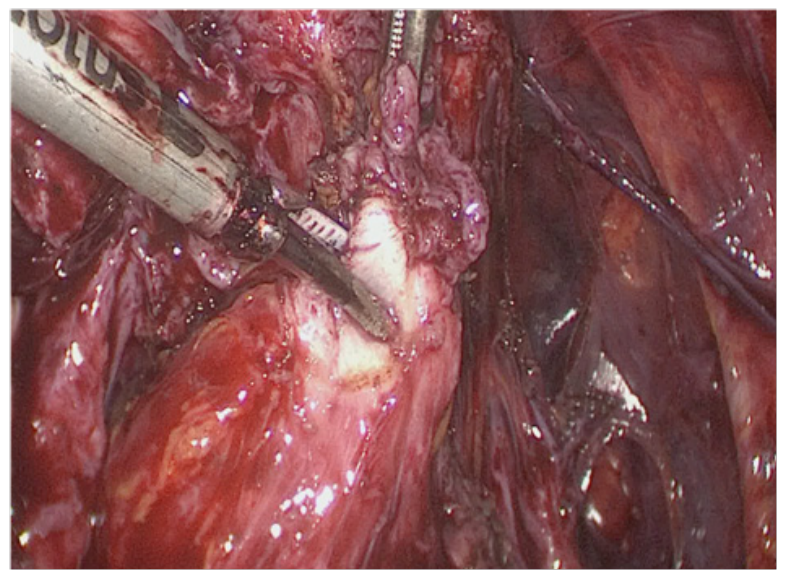

Figure 4 Distance between the border of the lesion and the ureteric orifice is $<2 \mathrm{~cm}$.

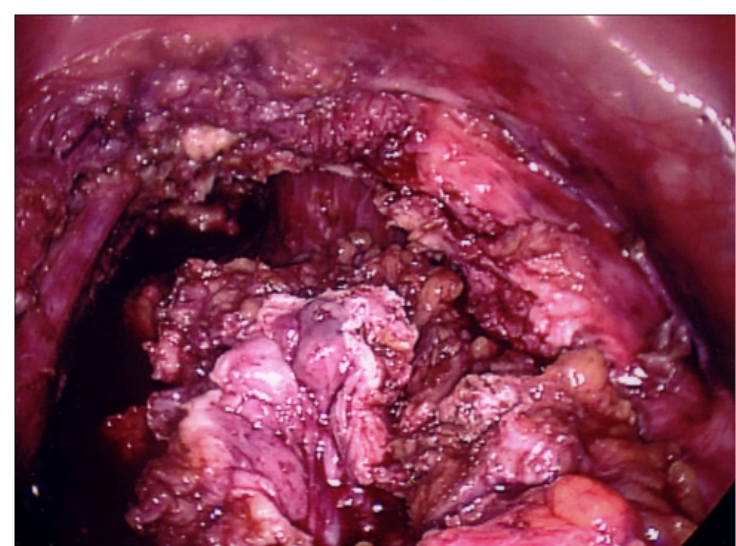

Figure 5 End-to-end anastomosis or re-implantation with/without a poses hitch.

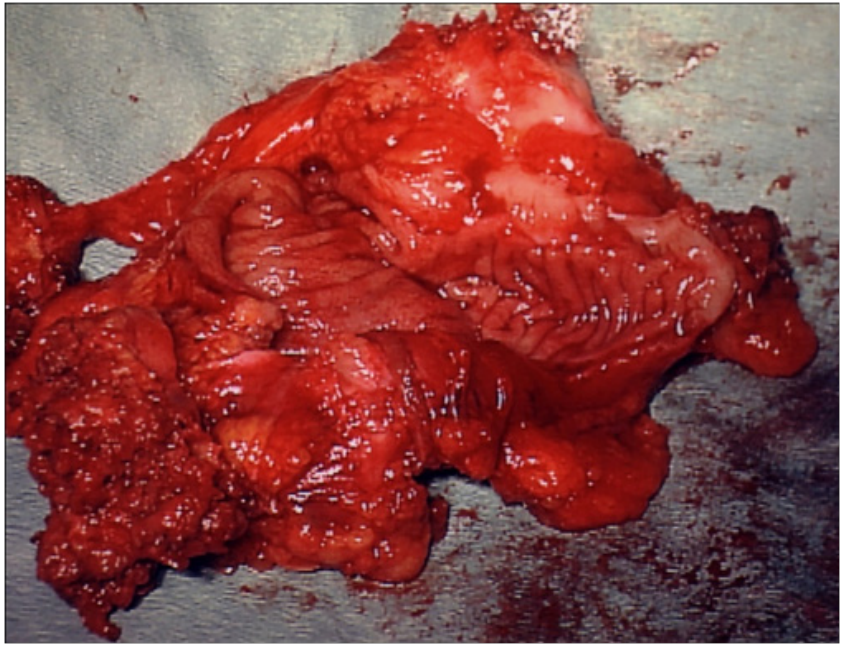

Figure 6 Rectal shaving is performed by excising a serosal disc of endometriosis off the rectal wall and overlying sutures placed to reinforce the excised area.

\section{Bowel endometriosis}

The exact prevalence of bowel endometriosis is unknown, although it is estimated that $5-12 \%$ of women with endometriosis will have bowel involvement, equating to 150,000 to 300,000 women in the UK. ${ }^{27,28}$ The rectum and recto-sigmoid tend to account for up to $93 \%$ of bowel lesions followed by the ileum, appendix and caecum. ${ }^{29}$ The depth of involvement can range from serosal to mucosal and lesions can be solitary or multiple. Symptoms vary, but most commonly include dyschezia, constipation, diarrhoea, bloating, passing of mucus per rectum and cyclical rectal bleeding. The management of disease involving the bowel remains a highly controversial and contentious issue. Medical treatment has been found to be largely inadequate for rectal disease. ${ }^{30,31}$ Surgical treatment and the complete excision of endometriosis appears to offer good long term symptomatic relief, ${ }^{32-34}$ however this may involve significant bowel surgery and the potential for major complications. Conversely less invasive surgery is associated with fewer complications, but incomplete resection carries a higher risk of disease recurrence and generally does not confer the long-term symptomatic benefits seen with complete resection. ${ }^{27}$ Two surgical approaches are generally employed, the more radical approach involving segmental bowel resection or the more conservative approach involving either rectal shaving or disc resection. Rectal shaving is performed by excising a serosal disc of endometriosis off the rectal wall and overlying sutures placed to reinforce the excised area (Figure 6). Disc resection involves removal of the endometriotic nodule by resecting a full thickness "disc" of bowel; this can be done using a circular stapler or excising the area and suturing the defect laparoscopically. The reported outcomes in the literature following disc resection are positive and a prospective analysis of 500 cases reported high conception rates following surgery with low major complication and recurrence rates. ${ }^{35}$ However there are limitations to this technique; it tends to be more suitable for lesions on the anterior surface of the bowel and small nodules involving less than $50 \%$ of the circumference of the rectal wall. ${ }^{28}$ The technique is generally not suitable for large rectal nodules $(>3 \mathrm{~cm})$ and in cases where there are multiple bowel lesions. In cases where large nodules are "shaved" there is invariably some residual disease left behind. Remordiga et al. ${ }^{36}$ demonstrated that at least one third of patients undergoing disc 
resection have persistent disease ${ }^{36}$ however controversy exists as to whether this is symptomatically significant. The current literature suggests recurrence rates are significantly higher for disc excision when compared to segmental resection. ${ }^{37}$ Segmental bowel resection involves resection of the pelvic endometriosis leaving the residual disease attached to the rectum and mobilisation of the rectal tube involving lateral and anterior retroperitoneal dissection. The bowel is then transected below the level of disease and to a clear margin above the proximal end of the disease. Primary anastomosis is then performed using a circular anastomosis gun and the integrity of the anastomosis checked by filling the pelvis with fluid and distending the rectum with air insufflated via a rigid sigmoidoscopy. If an air leak is visible, the anastomosis is inadequate and a defunctioning stoma is performed. Over the past 10 years, a large number of studies have shown significant improvements in pain and overall quality of life following radical surgery. ${ }^{32-34}$ However it can be associated with significant complications. Both minor and major complications have been reported following radical surgery including fistula formation $(0-14 \%),{ }^{27,32}$ anastomotic leaks $(2.2-8 \%)$ hemorrhage $(1-11 \%){ }^{38}$ infection (1-3\%), ${ }^{38}$ ureteric injury $(4.1 \%)^{32}$ and long term bladder (1$71 \%$ ) and bowel dysfunction (1-15\%). ${ }^{38}$ Kondo et al. reported a total major complication rate of $4.6 \%$ of patients undergoing any form of bowel surgery and $24,17.6$ and $6.7 \%$ for those undergoing segmental resection, disc resection and shaving respectively. ${ }^{27}$ Darai et al. ${ }^{15}$ reported a $12.6 \%$ major complication rate following laparoscopic segmental bowel resection. ${ }^{39}$ Long-term bladder and bowel dysfunction (urinary retention, tenesmus, frequency of defecation) are often the most disabling long-term complications encountered by patients following radical surgery. Rectal shaving certainly avoids the need for deep lateral rectal dissection and hence preserves the pelvic autonomic nerves and thus as expected fewer bladder and bowel complications are reported post-operatively, ${ }^{35}$ however once again this must be balanced again the risk of residual disease and recurrence. More recently nerve sparing rectal resection techniques have been described in the literature with identification and sparing of the hypo gastric and pelvic splanchnic nerves. This technique when compared to the standard technique appears to be associated with significantly less bladder/bowel and sexual dysfunction. ${ }^{40,41}$ Thus there is still no definitive consensus on which approach is the "best" technique to treat bowel endometriosis; ultimately the aim of treating DIE affecting the bowel should focus on the long-term resolution of bowel symptoms with the fewest complications. Detailed counseling and a multidisciplinary approach is key. Ultimately the surgical technique employed should be tailored to patient's symptoms, preoperative assessment and surgical findings. A balance must be made against suboptimal resection and symptom recurrence, and extensive surgical excision and the potential for major complications

\section{Use of robotic surgery for the treatment of DIE}

Recently the use of robotic surgery has increased in the treatment of DIE, however most of these studies are from outside the UK and the current recommendation from NICE is that a surgical robot should only be used in gynecology in a research setting. Almost all of the studies are observational case series with the largest published being a retrospective review of 164 patients with stage 4 endometriosis. ${ }^{42}$ This study described a lower conversion to laparotomy rate with the robotic approach $(0.6 \%$ vs. $10 \%)$. Another study by Dulemba et al. compared operative time ( 77 vs. $72 \mathrm{~min})$, blood loss ( 29 vs. $25 \mathrm{~mL}$ ) and complication rates $(1.1 \%$ vs. $0 \%)$ in robot-assisted and standard laparoscopy ${ }^{43}$ and found no significant differences. However they did note that the number of biopsies confirming endometriosis was higher in the robotic group $(80 \% \mathrm{vs} 56.8 \% \mathrm{P}<0.001)$ and hypothesised that robotic surgery, with its improved visual acuity, may have the ability to excise endometriosis more precisely. In the absence of any randomized case-control studies, it is difficult to recommend its usage in routine practice, however as its use becomes more prevalent in the UK there is a definite need for larger multi-centre trials.

\section{Fertility sparing surgery}

Women with confirmed endometriosis, who desire to conceive, should be referred to secondary care specialist services. Hormonal treatment is not an option in this group as this does not improve fertility and may cause further delays in surgical and assisted reproductive treatments that are known to potentially improve fertility. ${ }^{4}$ The benefit of surgical treatment to improve fertility in patients with endometriosis is well established in the literature by three Cochrane reviews ${ }^{44-46}$ and the European Society of Human Reproduction and Embryology (ESHRE) guidelines on endometriosis. ${ }^{4}$

In women with minimal to mild endometriosis, there is evidence that operative laparoscopy significantly improves chances of conception. ${ }^{45}$ It is suggested that where possible this could be done at the time of initial laparoscopy. What remains unclear to date is the comparative surgical effectiveness of the various treatment techniques. There is also evidence that laparoscopic surgical excision of moderate to severe endometriosis and adhesiolysis improves spontaneous pregnancy rates by as much as $57-69 \%$ (moderate endometriosis) and $52-68 \%$ (severe endometriosis). ${ }^{47,48}$ With no treatment spontaneous conception rates can remain as low as $0 \%$ in severe endometriosis and $30 \%$ in moderate endometriosis cases. ${ }^{49}$ For patients with endometriomas, excision of the endometrioma results in higher pregnancy rates when compared to drainage and electro-coagulation of the cyst. Both the techniques are known to deplete the ovarian reserves ${ }^{44}$ and this should be kept in mind and discussed with the patient before surgery, especially in cases of recurrent endometriomas. With the improvements in egg freezing technology, this might be a possible option for this group of patients in the future. For patients with rectovaginal disease complete excision of the disease including bowel resection and anastomosis may have higher spontaneous pregnancy rates when compared to limited excision of endometriosis without bowel resection, ${ }^{49}$ however the severity of symptoms rather than fertility should be the deciding factor when it comes to extensive resection.

\section{Conclusion}

Severe endometriosis is a complex condition requiring a multidisciplinary approach in a tertiary referral centre and early referral is essential. Full pre-operative planning including detailed imaging is vital to assess the full extent of the disease, give thorough counseling and plan appropriate surgical management. Radical excision of DIE involving both bladder and bowel appears to have good success rates with significant improvements in quality of life, improved fertility and low recurrence rates. However such extensive procedures carry both short and long-term risks that patients must be fully aware of before embarking down the aggressive surgical route. 


\section{Acknowledgements}

None.

\section{Conflict of interest}

The author declares no conflict of interest.

\section{References}

1. Ozkan S, Murk W, Arici A. Endometriosis and infertility: epidemiology and evidence-based treatments. Ann N Y Acad Sci. 2008;1127:92-100.

2. Culley L, Law C, Hudson N, et al. The social and psychological impact of endometriosis on women's lives: a critical narrative review. Hum Reprod Update. 2013;19(6):625-639.

3. Koninckx PR, Meuleman C, Demeyere S, et al. Suggestive evidence that pelvic endometriosis is a progressive disease, whereas deeply infiltrating endometriosis is associated with pelvic pain. Fertil Steril. 1991;55(4):759-765.

4. Dunselman GA, Vermeulen N, Becker C, et al. ESHRE guideline: management of women with endometriosis. Hum Reprod. 2014;29(3):400 412 .

5. Seracchioli R, Mabrouk M, Guerrini M, et al. Dyschezia and posterior deep infiltrating endometriosis: analysis of 360cases. J Minim Invasive Gynecol. 2008;15(6):695-699.

6. Perez-Utrilla Perez M, Aguilera Bazan A, Alonso Dorrego JM, et al. Urinary tract endometriosis: clinical, diagnostic, and therapeutic aspects. Urology. 2009;73(1):47-51

7. Ballard K, Lowton K, Wright J. What's the delay? A qualitative study of women's experiences of reaching a diagnosis of endometriosis. Fertil Steril. 2006;86(5):1296-1301.

8. Roman H, Carilho J, Da Costa C, et al. Computed tomography-based virtual colonoscopy in the assessment of bowel endometriosis: The surgeon's point of view. Gynecol Obstet Fertil. 2016;44(1):3-10.

9. Hudelist G, English J, Thomas AE, et al. Diagnostic accuracy of transvaginal ultrasound for non-invasive diagnosis of bowel endometriosis: systematic review and meta-analysis. Ultrasound Obstet Gynecol. 2011;37(3):257-263.

10. Bazot M, Bornier C, Dubernard G, et al. Accuracy of magnetic resonance imaging and rectal endoscopic sonography for the prediction of location of deep pelvic endometriosis. Human Reproduction. 2007;22(5):1457-1463.

11. Koninckx PR, Ussia A, Adamyan L, et al. Deep endometriosis: definition, diagnosis, and treatment. Fertil Steril. 2012;98(3):564-571.

12. Delpy R, Barthet M, Gasmi M, et al. Value of endorectal ultrasonography for diagnosing rectovaginal septal endometriosis infiltrating the rectum. Endoscop. 2005;37(4):357-361.

13. Vercellini P, Frontino G, Pietropaolo G, et al. Deep endometriosis: definition, pathogenesis, and clinical management. J Am Assoc Gynecol Laparosc. 2004;11(2):153-161.

14. Maccagnano C, Pellucchi F, Rocchini L, et al. Diagnosis and treatmen of bladder endometriosis: state of the art. Urol Int. 2012;89(3):249-258.

15. Bazot M, Darai E, Hourani R, et al. Deep pelvic endometriosis: MR imaging for diagnosis and prediction of extension of disease. Radiology. 2004;232(2):379-389.

16. Faccioli N, Manfredi R, Mainardi $\mathrm{P}$, et al. Barium enema evaluation of colonic involvement in endometriosis. AJR Am J Roentgenol. 2008;190(4):1050-1054.
17. Savelli L, Manuzzi L, Coe M, et al. Comparison of transvaginal sonography and double-contrast barium enema for diagnosing deep infiltrating endometriosis of the posterior compartment. Ultrasound Obstet Gynecol. 2011;38(4):466-471.

18. Savelli L, Manuzzi L, Pollastri P, et al. Diagnostic accuracy and potential limitations of transvaginal sonography for bladder endometriosis. Ultrasound Obstet Gynecol. 2009;34(5):595-600.

19. Bazot M, Thomassin I, Hourani R, et al. Diagnostic accuracy of transvaginal sonography for deep pelvic endometriosis. Ultrasound Obstet Gynecol. 2004;24(2):180-185.

20. Balleyguier C, Chapron C, Dubuisson JB, et al. Comparison of magnetic resonance imaging and transvaginal ultrasonography in diagnosing bladder endometriosis. J Am Assoc Gynecol Laparosc. 2002;9(1):15-23.

21. Seracchioli R, Mabrouk M, Montanari G, et al. Conservative laparoscopic management of urinary tract endometriosis (UTE): surgical outcome and long-term follow-up. Fertil Steril. 2010;94(3):856-861.

22. Chou MT, Wang CJ, Lien RC. Prophylactic ureteral catheterization in gynecologic surgery: a 12-year randomized trial in a community hospital. International Urogynecology Journal. 2009;20(6):689-693.

23. Nezhat C, Nezhat F, Nezhat CH, et al. Urinary tract endometriosis treated by laparoscopy. Fertil Steril. 1996;66(6):920-924.

24. Donnez J, Nisolle M, Squifflet J. Ureteral endometriosis: a complication of rectovaginal endometriotic (adenomyotic) nodules. Fertil Steril. 2002;77(1):32-37.

25. Seracchioli R, Manuzzi L, Mabrouk M, et al. A multidisciplinary, minimally invasive approach for complicated deep infiltrating endometriosis. Fertil Steril. 2010;93(3):1007e1-3.

26. Jadoul P, Feyaerts A, Squifflet J, et al. Combined laparoscopic and vaginal approach for nephrectomy, ureterectomy, and removal of a large rectovaginal endometriotic nodule causing loss of renal function. J Minim Invasive Gynecol. 2007;14(2):256-259.

27. Kondo W, Bourdel N, Tamburro S, et al. Complications after surgery for deeply infiltrating pelvic endometriosis. Bjog. 2011;118(3):292-298.

28. Cutner A, Vyas S. Laparoscopic surgery for benign gynaecology. London: RCOG Press; 2011

29. Milone M, Vignali A, Milone F, et al. Colorectal resection in deep pelvic endometriosis: Surgical technique and post-operative complications. World Journal of Gastroenterology. 2015;21(47):13345-13351.

30. Fedele L, Bianchi S, Zanconato G, et al. Gonadotropin-releasing hormone agonist treatment for endometriosis of the rectovaginal septum. Am J Obstet Gynecol. 2000;183(6):1462-1467.

31. Busacca M, Somigliana E, Bianchi S, et al. Post-operative GnRH analogue treatment after conservative surgery for symptomatic endometriosis stage III-IV: a randomized controlled trial. Hum Reprod. 2001;16(11):23992402

32. English J, Sajid MS, Lo J, et al. Limited segmental rectal resection in the treatment of deeply infiltrating rectal endometriosis: 10 years' experience from a tertiary referral unit. Gastroenterol Rep (Oxf). 2014;2(4):288-294.

33. Ford J, English J, Miles WA, et al. Pain, quality of life and complications following the radical resection of rectovaginal endometriosis. Bjog. 2004;111(4):353-356

34. Dubernard G, Piketty M, Rouzier R, et al. Quality of life after laparoscopic colorectal resection for endometriosis. Hum Reprod. 2006;21(5):12431247 
35. Donnez J, Squifflet J. Complications, pregnancy and recurrence in a prospective series of 500 patients operated on by the shaving technique for deep rectovaginal endometriotic nodules. Hum Reprod. 2010;25(8):19491958.

36. Remorgida V, Ragni N, Ferrero S, et al. How complete is full thickness disc resection of bowel endometriotic lesions? A prospective surgical and histological study. Hum Reprod. 2005;20(8):2317-2320.

37. Brouwer R, Woods RJ. Rectal endometriosis: results of radical excision and review of published work. ANZ J Surg. 2007;77(7):562-571.

38. Darai E, Ackerman G, Bazot M, et al. Laparoscopic segmental colorectal resection for endometriosis: limits and complications. Surg Endosc. 2007;21(9):1572-1577.

39. Ceccaroni M, Clarizia R, Bruni F, et al. Nerve-sparing laparoscopic eradication of deep endometriosis with segmental rectal and parametrial resection: the Negrar method. A single-center, prospective, clinical trial. Surg Endosc. 2012;26(7):2029-2045.

40. Kavallaris A, Banz C, Chalvatzas N, et al. Laparoscopic nerve-sparing surgery of deep infiltrating endometriosis: description of the technique and patients' outcome. Arch Gynecol Obstet. 2011;84(1):131-135.

41. Collinet P, Leguevaque P, Neme RM, et al. Robot-assisted laparoscopy for deep infiltrating endometriosis: international multicentric retrospective study. Surg Endosc. 2014;28(8):2474-2479.

42. Dulemba JF, Pelzel C, Hubert HB. Retrospective analysis of robot-assisted versus standard laparoscopy in the treatment of pelvic pain indicative of endometriosis. Journal of Robotic Surgery. 2012;7(2):163-169.
43. Hart RJ, Hickey M, Maouris P, et al. Excisional surgery versus ablative surgery for ovarian endometriomata. Cochrane Database Syst Rev. 2008;2008(2):Cd004992.

44. Jacobson TZ, Duffy JM, Barlow D, et al. Laparoscopic surgery for subfertility associated with endometriosis. Cochrane Database Syst Rev. 2010;(1):Cd001398.

45. Duffy JM, Arambage K, Correa FJ, et al. Laparoscopic surgery for endometriosis. Cochrane Database Syst Rev. 2014;4:Cd011031.

46. Nezhat C, Crowgey S, Nezhat F. Videolaseroscopy for the treatment of endometriosis associated with infertility. Fertil Steril. 1989;51(2):237240 .

47. Vercellini P, Pietropaolo G, De Giorgi O, et al. Reproductive performance in infertile women with rectovaginal endometriosis: is surgery worthwhile? Am J Obstet Gynecol. 2006;195(5):1303-1310.

48. Olive DL, Stohs GF, Metzger DA, et al. Expectant management and hydrotubations in the treatment of endometriosis-associated infertility. Fertil Steril. 1985;44(1):35-41.

49. Stepniewska A, Pomini P, Guerriero M, et al. Colorectal endometriosis: benefits of long-term follow-up in patients who underwent laparoscopic surgery. Fertil Steril. 2010;93(7):2444-2446. 